Table 1. Main differences in both groups

\begin{tabular}{lcccc}
\hline & $\begin{array}{c}\text { Total } \\
\mathrm{N}=112\end{array}$ & $\begin{array}{c}\text { APS } \\
\mathrm{N}=57(50,9 \%)\end{array}$ & $\begin{array}{c}\text { TRU+APS } \\
\mathrm{N}=55(49,1 \%)\end{array}$ & $\mathrm{P}$ \\
\hline Age & $48,3 \pm 13,7$ & $46,9 \pm 14,3$ & $49,7 \pm 13,2$ & 0,2187 \\
Gender & $96(85,7 \%)$ & $45(78,9 \%)$ & $51(92,7 \%)$ & 0,0698 \\
APS duration (months) & $72[24-144]$ & $48[13-120]$ & $80[31-156]$ & 0,2329 \\
Venous thrombosis & $61(54,5 \%)$ & $25(43,9 \%)$ & $36(65,4 \%)$ & $\mathbf{0 , 0 2 1 8}$ \\
Arterial thrombosis & $63(56,2 \%)$ & $30(52,6 \%)$ & $33(60,0 \%)$ & 0,4320 \\
Thrombosis all & $97(86,6 \%)$ & $47(82,5 \%)$ & $50(90,9 \%)$ & 0,1891 \\
livedo reticularis & $53(47,3 \%)$ & $41(71,9 \%)$ & $12(21,8 \%)$ & $<0,0001$ \\
EEG changes & $18(16,1 \%)$ & $5(8,8 \%)$ & $13(23,6 \%)$ & 0,0323 \\
Epilepsy & $20(17,9 \%)$ & $8(14,0 \%)$ & $12(21,8 \%)$ & 0,2823 \\
\hline
\end{tabular}

leading to this feature can be distinct between this two conditions. Additionally, it appears to be more reasonable to add thrombocytopenia to clinical criteria of APS, because its frequency does not depend on SLE presence.

Disclosure of Interest: None declared

DOI: 10.1136/annrheumdis-2017-eular.1736

\section{AB0497 SIX CASES OF MACROPHAGE ACTIVATION SYNDROME AS PRESENTING MANIFESTATION OF SYSTEMIC LUPUS ERYTHEMATOSUS}

F. Dall'ara ${ }^{1}$, I. Cavazzana ${ }^{2}$, M. Frassi ${ }^{2}$, M. Taraborelli ${ }^{2}$, M. Fredi $^{2}$ F. Franceschini ${ }^{2}$, L. Andreoli ${ }^{1}$, A. Tincani ${ }^{1}$, P. Airò ${ }^{2} .{ }^{1}$ Rheumatology and Clinical Immunology, University of Brescia - Dscs; ${ }^{2}$ Rheumatology and Clinical Immunology, Spedali Civili of Brescia, Brescia, Italy

Background: Macrophage Activation Syndrome (MAS) is a life-threatening syndrome characterized by excessive immune activation. It can be triggered by conditions affecting immune homeostasis, such as infections, malignancies and rheumatologic disorders, including Systemic Lupus Erythematosus (SLE). In previous studies, prevalence of MAS among SLE patients ranged from $0.9 \%$ to $4.6 \%$.

Objectives: To describe the presentation and treatment of both MAS and SLE in patients with both syndromes.

Methods: Monocentric retrospective evaluation: patients with MAS according to HLH classification criteria were identified in our cohort of SLE patients (classified according to ACR and SLICC criteria)followed for at least 1 year between 1972 and 2014

Results: Among 511 patients with SLE (mean age at diagnosis:31 years \pm 2 ), 6 patients $(1.2 \%)$ with MAS were identified (all female). Their main clinical and laboratory features are reported in table 1.Median HLH score was 226.5 (IQR 204-254), with a probability of having MAS of $96 \%$.In all cases MAS happened simultaneously to the onset of SLE.Median age at diagnosis was 31.5 years, median SLEDAI was 12.All patients had fever above $38^{\circ} \mathrm{C}$, lymphadenopathy, hematological involvement,and high titer ANA positivity. Workup for infections and malignancies was negative in all cases.All patients were treated with corticosteroids $(100 \%$ received intravenous immunoglobulin pulse of methylprednisolone);concomitant medications were: cyclosporin A in $83 \%$, IVIG in $67 \%$, granulocyte colony-

Table 1: main clinical and laboratory features at diagnosis of SLE and MAS

\begin{tabular}{|c|c|c|c|}
\hline \multicolumn{2}{|c|}{ Clinical features of MAS n (\%) } & \multicolumn{2}{|c|}{ SLE ACR classification criteria } \\
\hline $\begin{array}{l}\text { Fever } \\
\text { Hemorrhages } \\
\text { CNS dysfunction } \\
\text { Lymphadenopathy } \\
\text { Hepatomegaly } \\
\text { Splenomegaly }\end{array}$ & \begin{tabular}{|l|}
$6(100 \%)$ \\
$1(17 \%)$ \\
$0(0 \%)$ \\
$6(100 \%)$ \\
$4(67 \%)$ \\
$4(67 \%)$ \\
\end{tabular} & $\begin{array}{l}\text { §Malar rash/Oral } \\
\text { ulcers/Photosensitivity/ } \\
\text { LED } \\
\text { Arthritis } \\
\text { Nephritis } \\
\text { Serositis } \\
\text { CNS disease } \\
\text { Haematological } \\
\text { involvement }\end{array}$ & $\begin{array}{l}5(83 \%) \\
2(33 \%) \\
0 \\
3(50 \%) \\
1(17 \%) \\
6(100 \%)\end{array}$ \\
\hline \multicolumn{2}{|c|}{ MAS Laboratory Parameters median (IQR) } & \multicolumn{2}{|l|}{ Autoantibodies n ( $\%)$} \\
\hline $\begin{array}{l}\text { WBC }\left(\times 10^{2} / \mathrm{hL}\right) \\
\text { Neutrophils }\left(\mathrm{x} 10^{3} / \mathrm{mL}\right) \\
\text { HGB }(\mathrm{g} / \mathrm{dL}) \\
\text { PLT }\left(\mathrm{x} 10^{3} / \mathrm{uL}\right) \\
\text { AST }(\mathrm{U} / \mathrm{L}) \\
\text { ALT (U/L) } \\
\text { LDH (U/L) } \\
\text { Ferritin }(\mu \mathrm{g} / \mathrm{L}) \\
\text { Fibrinogen }(\mathrm{mg} / \mathrm{dL}) \\
\text { Triglycerides (mg/dl) } \\
\text { ESR (mm) } \\
\text { CRP (mg/dl) }\end{array}$ & \begin{tabular}{|l|}
$2.1(1.8-2.3)$ \\
$1.5(0.87-1.8)$ \\
$7.6(7-8,2)$ \\
$134(72-142)$ \\
$250(166-402)$ \\
$111(92-135)$ \\
$769(535-915)$ \\
$4607(1897-31533)$ \\
$97(35-170)$ \\
$511(317-605)$ \\
$17.5(9-24)$ \\
$37(10-49)$
\end{tabular} & $\begin{array}{l}\text { ANA } \\
\text { anti-ds DNA } \\
\text { anti-RNP } \\
\text { anti-Ro } \\
\text { anti-Sm } \\
\text { antiphospholipid } \\
\text { Ab/LA } \\
\text { *Direct Coombs Test + } \\
\text { C3 mg/dl (n.v 80-160) } \\
\text { C4 mg/dl (n.v 10-40) }\end{array}$ & \begin{tabular}{|l}
$6(100 \%)$ \\
$5(83 \%)$ \\
$2(33 \%)$ \\
$1(17 \%)$ \\
$1(17 \%)$ \\
$3(50 \%)$ \\
\\
$5(100 \%)$ \\
$34(28-70)$ \\
$13(7-17)$
\end{tabular} \\
\hline
\end{tabular}

* this test was available only for 5 patients

§at least one of these criteria: malar rash; Oral ulcers; Photosensitivity; LED: Discoid Lupus Erythematosus

CNS: central nervous system; WBC white blood cells; HGB: hemoglobin; PLT: plateletAST: aspartate aminotrasferase; ALT: alanine aminotrasferase; LDH: lactate dehydrogenase; ESR: erytrocyte sedimentation rate; CRP: C-reactive protein; ANA antinuclear antibodies; anti-ds DNA: anti double stranded DNA antibodies; anti-RNP: anti-ribonucleoprotein antibodies; LA: Lupus anticoagulant: n.v.: normal values stimulating factor in $17 \%$, mycophenolate mofetil in $17 \%$, etoposide in $17 \%$ and plasma exchange in $17 \%$. Two patients required haemotransfusion.All cases required hospital admission, and 2 were admitted in intensive care unit. No death from MAS was observed (median follow up: 34.5 months;IQR25-48). One patient died 44 months after after MAS for pulmonary adenocarcinoma.Table 1:main clinical and laboratory features at diagnosis of SLE and MAS

Conclusions: MAS is a rare complication in our SLE cohort and can complicate the onset of SLE, but it seems to be a very uncommon manifestation during the course of the disease.Fever may be a red flag for possible MAS, particularly if temperature is persistently above $38^{\circ}$ in absence of signs and symptoms of underlying infection.In our series, all cases were treated successfully with immunosuppressive drugs and cytotoxic agents such as etoposide were used only in one case.

Disclosure of Interest: None declared

DOI: 10.1136/annrheumdis-2017-eular.6692

\section{AB0498 SOLUBLE CD14 (PRESEPSIN) AS A POTENTIAL BIOMARKER TO DISCRIMINATE INFECTION VS. ACTIVITY IN PATIENTS WITH SYSTEMIC LUPUS ERYTHEMATOSUS}

I. Posso-Osorio ${ }^{1}$, A. Echeverry ${ }^{1}$, D. Aguirre-Valencia ${ }^{1}$, G. Castaño ${ }^{2}$, G. Tobón ${ }^{1}$ ${ }^{1}$ Rheumatology; ${ }^{2}$ Laboratory of inmunology, Fundación Valle del Lili, CALI, Colombia

Background: Differentiation of Systemic Lupus Erythematosus (SLE) activity and infection in a febrile SLE patient become difficult, since initial clinical presentation may be similar. Several biological markers (including procalcitonin and CRP) have been evaluated, with discordant results. Soluble CD14 (SCD14), also called presepsin, is the receptor for lipopolysaccharide - lipopolysaccharide binding protein (LPS-LBP) complexes. CD14 could activate a series of signal transduction pathways and inflammatory cascades, and lead to systemic inflammatory responses.(1)

Objectives: The aim of this study is to evaluate the utility of SCD14 as a biomarker to differentiate infection vs. activity in SLE patients admitted with systemic inflammatory response (SIRS).

Methods: We included 11 patients with SLE (ACR criteria 1997) and SIRS (International conference 2001) admitted to the ER and/or ICU. The measurement of SCD14 in plasma by enzymatic immunoassay of chemiluminescence in vitro was performed to differentiate active SLE vs. infection. Infection was considered if a positive culture/PCR was obtained. Mann-Whitney test was used to evaluate the association of variables with infection.

Results: All patients were female; mean age 37.9 years. An infectious disease was confirmed in 5 cases ( 3 bacterial including urinary tract infection, pneumonia and bacteremia; 1 viral infection by Chikungunya virus and 1 fungal by histoplasma capsulatum). SCD14 was elevated in the infected SLE patients (median: 1005 $\mathrm{pg} / \mathrm{mL}-\mathrm{RIC}$ : 533-1415-) vs patients with lupus flare (median: $431.5 \mathrm{pg} / \mathrm{mL}$ - RIC: 369-579-) $(\mathrm{p}=0,04)$

Conclusions: High values of SCD14 levels seem to be useful to differentiate infections from activity in SLE patients with SIRS. More patients and further analysis are necessary to define the clinical use of this biomarker.

References:

[1] Zou Q, Wen W, Zhang X. Presepsin as a novel sepsis biomarker. World $\mathrm{J}$ Emerg Med [Internet]. Second Affiliated Hospital of Zhejiang University School of Medicine; 2014 Aug 22;5(1):16-9. Available from: http://www.ncbi. nlm.nih.gov/pmc/articles/PMC4129857.

Disclosure of Interest: None declared

DOI: 10.1136/annrheumdis-2017-eular.2495

\section{AB0499 TOTAL BODY WATER AND ITS CORRELATION WITH SICCA SYMPTOMS IN PRIMARY SJÖGREN'S SYNDROME}

G. Hernandez-Molina ${ }^{1}$, P. Almeda-Valdés ${ }^{2}$, G. López-Carrasco ${ }^{2}$,

M. Astudillo-Angel ${ }^{1}$, M. González-Acevedo ${ }^{2}$, V. Zamora-Legoff ${ }^{1}$,

P. Sánchez-Fernández ${ }^{2}$, C. Aguilar-Salinas ${ }^{2}$, I. Cruz Bautista ${ }^{2}$. 'Immunology and Rheumatology; ${ }^{2}$ Endocrinology Department, Instituto Nacional de Ciencias Medicas y Nutricion Salvador Zubiran, Mexico City, Mexico

Background: Patients with primary Sjögrens' syndrome (PSS) suffer from severe alterations in both the quality and quantity of saliva and tears. Body water represents around $50-55 \%$ of the body weight. Tears contain $98 \%$ of water and saliva $99.5 \%$

Objectives: To evaluate the percentage of total body water (TBW) among patients with PSS and to assess its correlation with sicca symptoms.

Methods: We included 85 patients with PSS and 85 historical non diabetic controls matched by gender, age ( \pm 3 years) and body mass index $\left( \pm 1 \mathrm{~kg} / \mathrm{m}^{2}\right)(\mathrm{BMI})$. We assessed the presence of sicca symptoms, Schirmer-I test, non-stimulated whole salivary flow (NSWSF) and ocular staining. We also evaluated the ocular and oral domains of the ESSPRI, a validated scale for symptoms (a higher score implies worst symptoms). We obtained the TBW percentage with a bioelectric impedance analysis (BIA-SECA-514, Hamburgo)

Results: $80 \%$ were women, mean age $54.8+13.7$ years and mean disease duration $11.5 \pm 7.52$ years. The percentage of TBW was similar among patients and controls (PSS $46.85 \pm 4.6$ vs. $46.9 \pm 4.5, p=0.88$ ). Among the patients, the 
TBW negatively correlated with age $(\rho=-0.25, \mathrm{p}=0.02)$, disease duration $(\rho=-0.30$, $p=0.005)$, BMI $(\rho=-0.78, p=0.001)$ and the ocular component of the ESSPRI $(\rho=-0.28, p=0.01)$, but not with the NSWSF or the ESSPRI oral component. When we compared the patients in the $25 \%$ percentile (group with the lowest $\%$ of water)

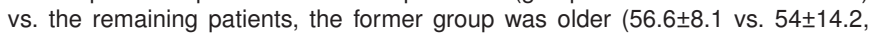
$p=0.02$ ), with longer disease duration $(12.4 \pm 5.9$ vs. $10.8 \pm 7.12, p=0.03)$, lower scores at the Schirmer test (1 (range $0-8)$ vs. 2 (range $0-9$ ), $p=0.01$ ), higher BMI $(31.1 \pm 5.1$ vs. $23.7 \pm 2.9, p=0.001)$ as well as with higher ESSPRI ocular domain scores $(8.3 \pm 1.4$ vs. $6.7 \pm 2.5, p=0.007)$. With the linear regression analysis, the variables that remained associated with the TBW were disease duration $(\beta-0.22$, $p=0.001)$, BMI $(\beta-0.76, p<0.001)$ and the ocular domain of the ESSPRI $(\beta-0.15$, $\mathrm{p}<0.001)$.

Conclusions: Patients with PSS had similar TBW percentage than controls. However among patients with PSS, the TBW had a negative correlation with the intensity of ocular symptoms independently of disease duration, age and BMI. References:

[1] Guyton, Arthur C. (2006). Textbook of Medical Physiology (11th ed.). Philadelphia: W.B. Elsevier Saunders. p. 295. ISBN 0-7216-0240-1.

Disclosure of Interest: None declared

DOI: 10.1136/annrheumdis-2017-eular.2388

\section{AB0500 TOLERABILITY, EFFICACY AND IMMUNOGENICITY OF 23-VALENT PNEUMOCOCCAL VACCINE IN SLE PATIENTS}

G.M. Tarasova, B.S. Belov, M.S. Sergeeva, S.K. Soloviev, E.A. Aseeva, N.G. Klukvina, T.V. Popkova, E.N. Alexandrova, A.A. Novikov, M.V. Cherkasova. V.A.Nasonova Research Institute of Rheumatology, Moscow, Russian Federation

Background: Concurrent infections turn out to be the second leading cause of death in systemic lupus erythematosus (SLE) pts after SLE per se. Immunization of SLE pts with pneumococcal vaccine is an important prophylactic approach to prevent severe lower respiratory tract (LRT) infections in SLE pts.

Objectives: To study the relevance of 23-valent pneumococcal vaccine for immunization of SLE pts.

Methods: The study included 30 SLE pts, 27 females, 3 males, aged $19-62$ y. Duration of follow up (FUP) was 12 months in 24 pts, and 7-10 months - in 6 pts. High disease activity at the time of immunization was documented in 1 patient, low activity - in 20 pts, moderate - in 4 pts, and remission - in 5. 29 pts were treated with glucocorticosteroids (GCs), 23 - with hydroxychloroquine, 14 pts - with cytostatic (CS) agents. Twelve pts were on biological diseasemodifying antirheumatic drugs (bDMARDs). One dose $(0,5 \mathrm{ml}$ ) of 23-valent polysaccharide pneumococcal vaccine was administered subcutaneously. The duration of FUP was 7-12 months. Control visits were scheduled as follows: at baseline (Visit 1), at 1st, 3rd, and 12th months (Visit 4) after immunization. Standard clinical examination and lab tests, including blood immunology, were performed at each visit. Vaccine immunogenicity was evaluated based on the level of serum antibodies (AT) to Streptococcus pneumoniae capsular polysaccharide (VaccZymeTM PCP Ig 2 panels (The Binding Site Ltd, Birmingham, UK)) - 4 times during 1 year.

Results: No post-immunization complications were seen in $11(36,7 \%)$ pts, local reactions of varying intensity lasting from 2 to 7 days were documented in $18(60 \%)$ pts. One patient $(3,3 \%)$ developed the local type III hypersensitivity reaction known as Arthus phenomenon. All symptoms subsided within 7 days after administration of antihistaminic agents and local GCs. Not a single vaccinationrelated SLE exacerbation episode was documented in 24 pts during the FUP. Significant ( $\geq 2$-fold vs baseline) increase of serum AT levels to $S$. pneumoniae polysaccharide was observed during the FUP (Table).

In $10(41,7 \%)$ out of 24 pts ("non-responders") more than 2-fold increase of antiS. pneumoniae ATs was not achieved by 12th month of FUP. Among them 7 $(70 \%)$ pts were receiving bDMARDs. $4(28,6 \%)$ out of 14 "responders " were also treated with bDMARDs.

Non-severe pneumonia was documented in 2 out of 24 pts within 1 year after vaccination; both cases successfully resolved after 7 - and 5-days of oral antibiotic treatment in an out-patient setting. Both pts had episodes of pneumonia in past medical history. One of them had SLE-induced interstitial lung disease. This patient was treated with GCs, mycophenolate mofetil, and rituximab, no post-vaccination response was documented. The second patient had two previous pneumonia episodes, she demonstrated 4-5-fold increase in anti- S. pneumoniae AT titre; her therapy included GCs $(10 \mathrm{mg} /$ day $)$ and hydroxychloroquine. There were no clinical or radiological symptoms of pneumonia in remaining 22 pts during the whole FUP.

\begin{tabular}{|c|c|c|c|c|}
\hline Visit & Visit 1 (baseline) & Visit 2 (1 mo $)$ & Visit $3(3 \mathrm{mo})$ & Visit 4 (12 mo) \\
\hline $\begin{array}{c}\text { AT } \\
\text { concentration } \\
\text { Mel }\end{array}$ & $\begin{array}{c}97,4^{*} \\
(68,0 ; 128,1)\end{array}$ & $\begin{array}{c}407,8^{b} \\
(231,2 ; 488,8)\end{array}$ & $\begin{array}{c}301,8 \\
(164,3 ; 424,6)\end{array}$ & $\begin{array}{c}265,5^{d} \\
(120,0 ; 438,7)\end{array}$ \\
\hline & & b-a, $p=0,00006$ & $c-2, p=0,00008$ & $\mathrm{~d}-\mathrm{a}, p=0,002$ \\
\hline
\end{tabular}

Conclusions: Obtained results are indicative of good tolerability, safety and immunogenicity of 23-valent pneumococcal vaccine in SLE pts. Further studies are necessary for more comprehensive evaluation of vaccine clinical efficacy.

Disclosure of Interest: None declared

DOI: 10.1136/annrheumdis-2017-eular.2347

\section{AB0501 THE ASSOCIATION BETWEEN GLUCOCORTICOIDS AND DAMAGE ACCRUAL IN PATIENTS WITH SLE USING GLUCOCORTICOID FOR LONG-TERM}

G. Wang, X. Li, Z. Dou, W. Wang, R. Liu. Rheumatology and Immunology, Anhui Provincial Hospital Affiliated to Anhui Medical University, Hefei, China

Background: Systemic lupus erythematosus (SLE) is a chronic systemic autoimmune disease characterized by a relapsing-remitting course. Long-term prognosis of SLE patients remains poor [1]. Due to the effect of potent anti-inflammatory and immunosuppressive, glucocorticoids (GCs) remain the cornerstone of treatment in SLE. However, GCs produce several adverse reactions, most are time and dose dependent, limiting their clinical usefulness. Increased longevity with prolonged exposure to GCs and inflammatory insults might contribute to organ damage accrual, which retards further improvement of survival in these patients [2]. Assessment the extent of organ damage caused by SLE has been considered an important part of the assessment of prognosis. The Systemic Lupus International Collaborating Clinics/American College of Rheumatology (SLICC/ACR) Damage Index (SDI) is a validated instrument designed to measure irreversible damage resulting from SLE disease activity and its treatment. The study of damage accrual in patients with SLE caused by the long-term treatment of GCs is still not clear.In a large SLE cohort, followed prospectively, we determined to investigate the association between damage accrual with GCs, both cumulative prednisone dose and high-dose prednisone. The results of our study could shed more light on the risk/benefit ratio of GCs in long-term maintenance treatment with SLE patients.

Objectives: To evaluate the association between long-term glucocorticoids use and damage accrual in patients with systemic lupus erythematosus.

Methods: Medical records of 535 SLE patients from Department of Rheumatology and immunology of Anhui Provincial Hospital were reviewed. 512 patients were femal. The cohort's mean age was $38.27 \pm 12.84$ years with mean disease duration of $7.33 \pm 5.75$ years. Their Systemic Lupus International Collaborating Clinics/American College of Rheumatology Damage Index (SDI) scores were noted. (1) Univariate analysis and multivariable regression analysis were performed to determine factors associated with SDI. (2) Analysis were also performed to determine damage associated with cumulative prednisone dose and high-dose of prednisone (exposure to prednisone at a dosage of $60 \mathrm{mg} /$ day for $1 \mathrm{month}$ ). Results: (1) Among 535 patients in our cohort only 5 paitents $(0.9 \%)$ had never been treated with glucocorticoids. A total of 192 patients $(35.9 \%)$ had been treated with high dose of prednisone. In addition, $86.9 \%$ of patients had been treated with hydroxychloroquine. (2) The highest organ damage was musculoskeletal $(n=79,14.8 \%)$, followed by skin damage $(n=35,6.5 \%)$ and renal $(n=28,5.2 \%)$. Ninety patients were diagnosed with hypertension. (3) SDI scores were associated with age of onset, exposure to high-dose prednisone, hypertension.(4) Cumulative prednisone dose was associated with osteoporosis, osteonecrosis and hypertension; exposure to high-dose prednisone was associated with osteonecrosis, lupus nephritis and hypertension.

Conclusions: Long-term taking prednisone predicted damage accrual. The most common damage was osteoporosis, osteonecrosis and hypertension.

References:

[1] Lopez R, Davidson JE, Beeby MD, et al. Lupus disease activity and the risk of subsequent organ damage and mortality in a large lupus cohort[J]. Rheumatology. 2012,51(3):491-8.

[2] Mak A, Isenberg DA, Lau CS. Global trends, potential mechanisms and early detection of organ damage in SLE[J]. Nature reviews Rheumatology. 2013,9(5):301-10.

Disclosure of Interest: None declared

DOI: 10.1136/annrheumdis-2017-eular.3931

\section{AB0502 STRONG REDUCTION OF ANTI-MÜLLERIAN HORMONE IN SYSTEMIC LUPUS ERYTHEMATOSUS WOMAN OF REPRODUCTIVE AGE}

C. Li, J. Xie, X. Wang, X. Liu, G. Chen. Division of Rheumatology and Clinical Immunology, the First Affiliated Hospital of Guangzhou University of Chinese Medicine, Guangzhou, China

Background: Systemic lupus erythematosus (SLE) is a clinically autoimmune disease characterized by production of autoantibodies and immune complex deposition. That induces multiple organ damages such as nephritis, pneumonitis and central nervous system (CNS) lupus et al. Moreover, SLE which mainly occurs in reproductive woman could threaten ovarian function. In recent years, ovarian reserve dysfunction in SLE are attracting increasing attentions. Especially cyclophosphamide (CYC) therapy was already well known as a higher risk to result in premature ovarian failure (POF) 1,2. However, few research has been performed in association between SLE itself and ovarian reserve. POF which is a critical cause of secondary amenorrhea and infertility still has not been treated effectively until now. Accordingly, an early precaution is important to make an informed decision about impaired ovarian reserve in SLE.

Objectives: To study the association between systemic lupus erythematosus (SLE) itself and strongly reduced Anti-Müllerian Hormone (AMH) values.

Methods: SLE women during reproductive ages 18-40 years were recruited compared with age-matched healthy controls (HC). AMH Levels and its relationship to clinical parameters and disease activity were investigated. 